\title{
ALGO EN COMÚN
}

Carlos E. Rendón E. (*) $^{*}$

“...Allí sugiere que la historia debería ser contada en primer lugar por sus protagonistas y sólo después por los especialistas; que la historia, antes de convertirse en densos volúmenes, sea elaborada primero como cuento, casi, se diría, como chismorreo de vecinos, en esas tardes largas y espaciosas en que las gentes comunes gozan amonedando en palabras los dramas y las maravillas del pasado y del presente"

William Ospina, Colombia en el planeta

¿Qué desea contar? ¿Qué quiere aprender? ¿Qué quiere enseñar?, son algunas de la preguntas y cuestionamientos que nos debemos hacer cuando se desea abortar temas socioculturales y patrimoniales. Patrimonio es un tema tan amplio, pero es justamente ahí donde radica el encanto pues no debemos pretender construir corolarios o modelos que estandaricen las individualidades; sino que debemos actuar como un $\mathrm{ADN}$ que tiene propósitos estructurales y regulan el uso de la información genética, que se debe traducir para poder funcionar pues existen multitudes y es característico de cada especie, símil a esta definición se debe abordar nuestro tema de interés representado por el amplio campo del que hacer del arte y la cultura tomado como escenario los territorios, como razón de ser el sujeto y su comunidad, como vehículo para llegar a ello el patrimonio, permitiendo acercar de manera biunívoca dichos patrimonios a la comunidad y la comunidad a los patrimonios. Es lograr comprender y aprender con todos los sentidos en justo equilibrio, es transmitir como una especie de rizoma.

Éste relato forma parte de las experiencias del macroproceso llamado Museo y Territorios, por más de 10 años en el Museo de Antioquia en Medellín - Colombia, es una invitación a corazón abierto a que nos atrevamos hacer las cosas de manera diferente. Todo ello con el propósito de superar los paradigmas que poseemos y el afán de protagonismo que es tan común y nocivo en este tipo de acciones. Se trata de nombrar las cosas de otra forma para salir del espacio de confort que anquilosa tanto nuestras áreas misionales y apostar por la construcción colectiva del conocimiento. Esto nos obliga a bajar los discursos hegemónicos, ser más cercanos e incluyentes a nuestras audiencias y comunidad de entorno. Las acciones aquí descritas son procesos de apropiación en contextos y localidades específicas de la ciudad de Medellín, Colombia y del territorio nacional, donde hemos logrado transitar y aportar a la solución de problemas de base social, pues albergan iniciativas para definir territorios de conocimiento que generan aprendizajes,

\footnotetext{
(*) Organización Plan C. E-mail: carlosedwinrendon@ gmail.com.
} 
reconocimiento de su entorno y del otro, donde lo más importante es la colaboración, la valoración, el intercambio y el compartir.

\section{EL PATRIMONIO CERCANO}

El arte y la cultura siempre serán un excelente escenario para aportar de manera directa a la construcción de ciudadanía, para consolidar nuestras identidades y patrimonios, pero esto requiere, sin duda, una apuesta política clara para ponerla en marcha (ORTEGA Y GASSET, 1956). También es poseer unas incertidumbres acerca del rol que debe cumplir el arte y la cultura en la comunidad, es estar dispuesto a bajar el discurso hegemónico y anquilosado que poseen las instituciones. Se trata de jugarnos la alternativa de la construcción colectiva del conocimiento, es afrontar el reto con un equipo profesional con humildad intelectual, es apostarle al arte y la cultura como un medio de trasformación social. Esto implica colocar el arte y al patrimonio de manera cercana a las personas; tal propósito no se alcanza en distancias cortas, a menudo el camino es largo y atraviesa por muchos y variados experimentos (FISCHER, 1964).

Al momento de abordar el territorio para iniciar los procesos socioculturales que dialogue de manera directa a las necesidades de las comunidades, de debe determinar en qué proposición nos deseamos parar cuando hablamos de trabajo comunitario, pues estamos en todo el derecho de trabajar: para la comunidad, por la comunidad, con la comunidad o en comunidad. La experiencia del excelente equipo de trabajo, colaboradores y amigos de viaje en el Museo de Antioquia se consolida en las preposiciones con y en. En estos escenarios se puede generar un verdadero acercamiento, democratización, accesibilidad, participación. El pedagogo de museo debe “...tener la capacidad de generar proyectos que puedan ser concebidos como laboratorios de ideas interdisciplinarias, que toman en cuenta a los públicos y promueven su participación" (Sadurni, 2014, p.48). Para así generar sentido de pertenencia y, por ende, lograr la construcción de ciudadanía desde los trabajos comunitarios.

Esto implica siete premisas.

Primero: Los proyectos no se construyen desde el escritorio.

Segundo: La utilización de estrategias didácticas debe ser dinámicas y construidas directamente en campo, deben exigir permanente actualización, co-evaluación, inter-evaluación y auto-evaluación. El que ejerce la pedagogía siempre está buscando la forma de hacerse entender y que los demás comprendan, reflexionen y cuestionen dicha información. 
Tercero: Tratar a la comunidad con dignidad y respeto, lo cual se logra fundamentalmente con una escucha activa, donde las propuestas planteadas respondan a las necesidades del territorio, que se ajusten a las variables intrínsecas y extrínsecas que hacen que cada comunidad sea diferente la una de la otra, así pertenezcan a la misma región; por consiguiente las estrategias deben ser a la medida y no responder a un modelo genérico.

Cuarto: Desmontar el rol de educando y educadores y apostarles a las comunidades de aprendizaje, de esta manera se concibe la construcción colectiva del conocimiento.

Quinto: Los horarios de encuentro no son nuestros horarios de oficina, sino los de disponibilidad de las comunidades, el tiempo de ellos donde se logre la mayor participación.

Sexto: El planteamiento del objetivo general, los objetivos específicos, seguimiento e indicadores, se genera directamente con la comunidad participante. Investigación acción participativa debe ser el baluarte de trabajo.

Séptimo: Los logros alcanzados se deben sedimentar con la construcción de sentido de pertenencia frente a las acciones que se estén realizando para evolucionar del espectáculo, la tarea extramural o el asistencialismo, a una verdadera capacidad instalada en los territorios con autonomía, sentido de pertenencia y continuidad.

Reflexión, cuestionamiento y trabajo continuo en pro de éstas premisas permite compartir acciones que trasciende los límites del escritorio y los muros institucionales, lo cual nos hace más escucha, permeables, porosos, receptivos a lo cotidiano y las tradiciones territoriales. De esta forma, logramos comprender que el patrimonio es móvil, camina, puede ser espontáneo, variable; puede ser creado en la esquina, en la vereda, en los caminos, en las carreteras, en las fábricas, en las huertas, en la escuela, en el hogar, incluso en las empresas. Es la cultura presente en los barrios, en los lugares donde vive la gente, personas que por su marginalidad cultural veían el patrimonio como algo totalmente ajeno, indiferente. Sin embargo, al actuar con propuestas, acciones y procesos socioculturales claros que responden a los intereses concretos de las comunidades se logra otra forma de construcción colectiva de sentidos, de proyectos propios, de reconstrucción de lo público, valoración del patrimonio y el papel protagónico e indispensables del individuo en los temas artísticos culturales en el territorio.

Éstas premisas permiten consolidar la concepción de lo humano, vivencial y cotidiano, abordando no solo el concepto, el significado, el significante de lo llamado patrimonio, sino generando una impronta personal y contemporánea. Una arquitectura cultural en donde se piense más el afuera que el adentro, en donde el límite del escritorio se desdibuje para incluir los 
territorios, la voz del otro; en donde la salvaguarda del patrimonio muta hacia un patrimonio vivo y cambiante que se vincula directamente al cotidiano, a la construcción participativa y colectiva de la comunidad, al ritmo de la vida que no es estática sino que vibra, cambia y evoluciona constantemente.

Aquí el patrimonio adquiere un compromiso más proactivo no sólo de acompañamiento de una definición universal, de una reflexión generalizada o de una teoría referencial. Al mismo tiempo permite sacudir ese discurso rancio para adaptarse a las realidades sociales de nuestras comunidades, de nuestro entorno. Permitiendo otros discursos, otras dialécticas, otras lecturas y una aproximación más viva a la comunidad, más propias, efectivas, donde cumpla una función comunicacional proactiva y cercana a los territorios. Lo cual otorga una comprensión de la historia, no como algo pasado sino como algo presente, del ahora. Una historia que se escribe a diario, viva, logrando un verdadero nivel de pertenencia entre el individuo y la concepción patrimonial, un patrimonio como herramienta de transformación social.

Por ello debemos construir un nuevo tesauro donde no busquemos salas de exposición sino espacios de hospitalidad, no realicemos inauguraciones sino festivales de la memoria, no convoquemos a talleres sino espacios de encuentro, no busquemos apoyo logístico sino compañeros de viaje, no hablemos de alumnos sino de comunidad de aprendizaje, no realicemos salidas de campo sino entradas a territorio abierto, no realicemos evaluaciones sino valoraciones, entre otros. Es de común acuerdo con la comunidad que se generan nuevas narrativas donde se concibe el patrimonio como algo más que artículos o leyes que los nombra, pasando a una construcción colectiva en donde yo (como individuo) también soy patrimonio. Donde se concibe y se construye las reflexiones y discusiones a partir de las propias definiciones, creencias y costumbres, como un ente vivo pensado con y en comunidad.

En este contexto se piensa el territorio como el resultado de una deliberación colectiva que se gesta y reinventa durante el desarrollo de los espacios de encuentro, reflexión y generación de más preguntas. Espacios donde las personas establecen lazos entre sí y cuando estas relaciones son duraderas se establecen redes (VARGAS ULATE, 2012). Aquí el desarrollo no llega desde la institución, sino de los mismos habitantes como artífices de las ideas. Los ejes temáticos a trabajar son necesarios tenerlos claros no para enseñarlos sino para abordarlos de manera conjunta, cuestionarlos, debatirlos, reflexionar a partir de ellos; al final de manera consensuada determinar si ellos se conservan, complementan, transforman, modifican o cambian. 
Las experiencias desarrolladas por Museo y Territorios durante 10 años en 18 municipios del Territorio Nacional, 124 municipios de Antioquia, 20 barrios de la ciudad de Medellín y 12 encuentros y procesos internacionales pretende ser una experiencia aportante y transformadora. Desde la cual se pueda establecer metodologías de trabajo que contribuyan a definir los espacios en compañía del otro, para sacar al ciudadano de a pie de los concepciones usuales, preconcebidas.

De igual modo favorezcan la participación activa de los ciudadanos y la integración de los conocimientos de los expertos y profesionales se conviertan en oportunidad para la creación colectiva. Este proceso permitiría el empoderamiento, sentido de pertenencia y acercamiento a ese patrimonio que de pronto no consideran tan cercano y propio.

Todo ello permite descentralizar los trabajos sobre cultura, arte, patrimonio, memoria, identidades y ser más generosos con el conocimiento para compartirlo y brindarlo. Dejando abierta la posibilidad para que cualquiera pueda hacer aportes, modificaciones, ajustes, transformaciones y lograr una verdadera apropiación de los conocimientos. Es poner a disposición de la comunidad saberes que alberga los estudiosos del tema y de ésta manera participar activamente de los espacios, contenidos, donde se debe ser modesto con el conocimiento y tener escucha activa para que la información se construya a partir de la colaboración horizontal, para que el conocimiento parta de la comunidad.

Por otra parte, sacudir y obligar a las instituciones que se hagan preguntas constantemente, reflexiones, debates y busquen espacios de encuentro diseñados para los diversos públicos; dejando de lado modelos y “...encontrar fórmulas específicas que surgidas en otros contextos nos tienten a repetir e implantar soluciones temporales, ya que sólo se rigen a eso: un tiempo y espacio determinado, sin asegurar nada más que su realización” (GALLEGOS, 2016, p. 4) para encontrar lenguajes propios desde lo contemporáneo.

Todos debemos estar comprometidos sin dejar de ser autónomos pero sí responsables del trabajo común. Es la manera de gestionar los propios procesos culturales, permitiendo nuevas formas de valorar el patrimonio y compartir las reflexiones, potenciando el desarrollo colaborativo. Así se pasa del modelo egoísta, egocéntrico y euro centrista a otro donde se reconoce que el conocimiento no es unidireccional sino de múltiples fuentes y que desde siempre ha sido colectivo. Esto permite un contacto más directo entre patrimonio, identidades, memoria, arte, cultura y comunidad; generando un conocimiento compartido, promoviendo ejes temáticos de interés común, eliminando las barreras, generando nuevos conocimientos de intercambio cultural. De modo que el territorio es el espacio propicio para fortalecer los procesos culturales implementando didácticas 
alternativas, disruptivas, potenciando las capacidades existentes de las comunidades y organizaciones sociales de base. De esta manera, la educación se nutre de la realidad del presente en pos de reconstruir continuamente sus métodos educativos y a la vez como una herramienta al servicio del cambio y la transformación de las sociedades (BRITO, 2008).

En consecuencia se proyecta el patrimonio como un verdadero territorio de cultura centrado entre el diálogo de organizaciones y una ciudadanía participante que interacciona con el arte visualizando las concepciones de patrimonio. Esto ocurre porque el arte es lo más enérgico que produce una época (APOLLINAIRE, 1994); lo cual proporciona un pretexto, una ocasión para la convergencia, el encuentro, el reconocimiento, para inventarnos, pensarnos, tener esperanzas, permitirnos soñar, imaginar, crear, evolucionar el día a día, explorando y compartiendo al pensarnos como parte de un todo que se llama patrimonio.

Finalizo con unas interrogantes, en homenaje a Paolo Freire y su Pedagogía de la Pregunta:

- Cuando decimos patrimonio hoy ¿qué es lo que realmente estamos nombrando? ¿No será el momento de intentar una nueva definición de la palabra patrimonio en nuestras sociedades latinoamericanas?

- ¿Son los museos preservadores del patrimonio de una sociedad o generadores y transformadores de esos patrimonios?

- ¿Para qué un museo en una ciudad en conflicto? ¿Qué aporta un encuentro artístico a una ciudad en transformación?

- Los museos en el mundo, tradicionalmente, se han hecho como un envoltorio para obras de marca, o para dar marca, reconocimiento, a esas obras. ¿Cómo son nuestras ciudades y sus contextos, ello se ve reflejado en nuestros museos? ¿Las colecciones que alberga los museo están en dialogo directo con el museo que somos o con el que queremos ser? ¿qué es lo que realmente consideramos como patrimonio?

"Es la hora de recostar las sillas en la puerta, y de empezar a contarla historia, antes de que lleguen los historiadores"

William Ospina, Colombia en el planeta 


\section{REFERENCIAS}

Apollinaire, G. Meditaciones estéticas. Los pintores cubistas. (1913). Madrid: Visor, 1994..

Brito, Z. Educación popular, cultura e identidad desde la perspectiva de Paulo Freire. In: GODOTTI, Moacir; GOMEZ, Margarita V.; MAFRA, Jason; ALENCAR, Anderson F. (Compiladores). Paulo Freire. Contribuciones para la pedagogía. Buenos Aires: Clacso, 2008. p. 29-45.

FISCHER, E. La necesidad de arte. La Habana: Editorial Unión, 1964.

GALLEGOS, C. El tiempo no es más fuerte: propuesta programa educativo y lineamientos para un macro-proceso en arte-educación. Cuenca, Ecuador: Fundación Municipal Bienal de Cuenca Disp.: <http://www.bienaldecuenca. org/menu/detalle/data/aWQ9MTY2. Consultado: 15 mayo 2016.

ORTEGA Y GASSET, J. La deshumanización del arte y otros ensayos de estética. Madrid: Alianza. 1991.

OSPINA, W. Colombia en el Planeta. Medellín: Imprenta Departamental de Antioquia, 2001.

SADURNI, N. Curaduría educativa:¿democratizar el espacio o ejercer el poder?. Nierika. Revista de Estudios de Arte. D.F. México, a. 3, n. 6, p. 35-48, jul.-dic. 2014. Disp.: <http://revistas.ibero.mx/arte/uploads/volumenes/6/pdf/ NIERIKA-NUM6.pdf>. Consultado: 10 abril 2016.

VARGAS ULATE, G. Espacio y Territorio en el Análisis Geográfico. Revista Reflexiones, Costa Rica, v. 91, n. 1, p. 313-326, 2012. Disp.: <http://www.redalyc.org/pdf/729/72923937025.pdf>. Consultado: 25 abril 2016. 


\section{RESUMEN}

La cultura debe ser una estrategia de diálogo entre el arte y la comunidad que permite consolidar a partir de la escucha activa esa concepción de lo humano, de lo vivencial y lo cotidiano. Abordando no solo conceptos patrimoniales, significados y significante de términos socioculturales; sino imprimiendo una impronta personal y contemporánea a la concepción de institución cultural y patrimonial. Con una arquitectura cultural en donde la institución se piense más en el afuera que en el adentro, en donde el límite de sus muros se desdibujen para incluir los territorios. Donde la salvaguarda del patrimonio muta hacia un patrimonio vivo y cambiante que se vincula directamente al cotidiano, a la construcción participativa y colectiva de la comunidad, al ritmo de la vida que no se queda estática sino que vibra y evoluciona constantemente. Es así como estos temas son relevantes a partir de construcción colectiva de sentido.

Palabras Clave: Patrimonio. Cultura. Diálogo.

\section{ALGO EM COMUM}

\section{RESUMO}

Cultura deve ser uma estratégia de diálogo entre a arte e a comunidade, que consolide, a partir da escuta ativa, essa concepção de ser humano, de experiência e de vida cotidiana. Dirigindo-se não só aos conceitos econômicos, seus significados e termos socioculturais significativos; mas a impressão de uma marca pessoal e contemporânea na concepção de instituição cultural e do patrimônio. Com uma arquitetura cultural onde a instituição pensa mais sobre o exterior do que $o$ interior, onde o limite de suas paredes possa somar para incluir os territórios. Onde se transforma proteção do patrimônio em uma vida, mudando a herança que está diretamente ligada à construção cotidiana, participativa e coletiva da comunidade. 0 ritmo de vida não é estático, mas vibra e está em constante evolução. Esta é a forma como estas questões são relevantes da construção coletiva de sentidos.

Palavras-chave: Patrimônio. Cultura. Diálogo.

\section{SOMETHING IN COMMON}

\section{ABSTRACT}

Culture should be a dialogue strategy between the art and the community to consolidate a conception of what is human from active listening, the existential and everyday life. Addressing not only heritage concepts, significant and socio-cultural meanings, but rather a personal printing in the design of contemporary the institution of culture and heritage. With a cultural architecture where the institution thinks itself more outside than inside, where the limit walls vanishes to include the territories. Where the safeguarding of static heritage shifts to the recognition of a living and changing heritage, that is directly linked to quotidian life, to the participatory and collective construction of the community, to the dynamic and changing movement of life which vibrates and evolves constantly. That is how these issues are relevant to the collective construction of meaning.

Keywords: Heritage. Cultural. Dialogue.

Submetido em: Ago. 2016

Aprovado em: Dez. 2016 